\title{
Molecular docking of fucoidan (a polysaccharide compound Turbinaria conoides) with carbonic anhydrase IX-A potent target molecule in breast cancer
}

\author{
Santhanam Revathi Chitra, Syed Ali Mohamed Yahoob*, Anuradha Venkatraman \\ PG \& Research Department of Biotechnology, Mohamed Sathak College of Arts and Science, Chennai, India.
}

\begin{tabular}{l}
\hline ARTICLE INFO \\
\hline Article history: \\
Received on: January 27, 2021 \\
Accepted on: May 09, 2021 \\
Available online: September 20, 2021 \\
\hline
\end{tabular}

Key words:

Carbonic anhydrase IX, docking, fucoidan, insilico, Molegro virtual docker, Turbinaria conoides

\begin{abstract}
Turbinaria conoides is a type of brown seaweed containing fucoidan, a complex polysaccharide. Fucoidan is known to have antiviral, antibacterial, antifungal, and anticancer activity. The current study is focused on the extraction of fucoidan from T. conoides and the extracted fucoidan (FE) was confirmed by high performance liquid chromatography using standard fucose and the anti-scavenging property of the FE was determined by ferric reducing antioxidant power assay (FRAP) and superoxide dismutase (SOD). The structure of fucoidan was retrieved from Pubchem (CID 92023653), and it was allowed to dock against the carbonic anhydrase IX enzyme retrieved from (PDB ID 2HKF) which is up-regulated by the cancer cells. FRAP and SOD showed significant anti-scavenging property compared to the standard and molecular docking analysis using the Molegro virtual docker (MVD 5.0) of fucoidan against the carbonic anhydrase IX (CA IX) showed significant binding affinity and hydrogen bond interactions with Molegro score -66.5818 with $8 \mathrm{H}$ bonds having an $\mathrm{H}$-bond score of -18.8865 . Fucoidan as a carbonic anhydrase IX inhibitor and consequently as a lead molecule against breast cancer can be further validated by suitable in vitro or in vivo investigation.
\end{abstract}

\section{INTRODUCTION}

Fucoidan is a polysaccharide that is present in the outer cell wall of the Turbinaria conoides; it is composed of fucose and sulfate molecules. The cell wall composition of the different seaweeds shows diversity in their structure and chemical composition. Generally, they have $(1,3)$ linkage of $\alpha$-L-fucopyranosyl residue or $(1,3)$ and $(1,4)$-linked $\alpha$-L- fucopyranosyl residues. In some cases, it may contain the sulphated galatofucans and mannopyranosyl units with fucose subunits in them. It is also composed of other simple sugars like galactose, D-xylose, D-glucose, D-mannose, D-glucuronic acid, and D-uronic acid in them [1]. In recent decades, brown seaweeds are found to be an important source having biologically active component in treating many disorders like anti-inflammatory [2], anticancer [3], immunomodulatory $[4,5]$, antiviral [6], and antioxidant activity [7]. Brown seaweeds

\footnotetext{
*Corresponding Author

Syed Ali Mohamed Yahoob, PG \& Research Department of Biotechnology, Mohamed Sathak College of Arts and Science, Chennai, India .

E-mail:syedmicro555@gmail.com
}

were found to contain potent antioxidants that are prominently accepted due to the absence of chemicals rather than synthetic antioxidants [8]. These brown seaweeds are found to possess potential secondary metabolites like fucoidans, terpenoid, phlorotanins, phenolic compounds, flavonoids, sterols, and glycolipids [9]. Free radicals otherwise called as reactive oxygen species (ROS) are one of the factors that are interlinked with many diseases and degenerative conditions [10,11]. Generally, body cells produce antioxidants to defend against oxidative stress apart from that antioxidants are synthesized from the food we consume (fruits and vegetables). Excessive production of ROS can cause damage to the biomolecules in the body this can be overcome by antioxidants naturally synthesized by the body cells [12]. Researchers have also found the antioxidant potential of natural sources against oxidative stress $[13,14]$. ROS is one of the main reasons for creating oxidative stress for the occurrence of many chronic infections, like asthma, inflammation, arthropathies, atherosclerosis, and cancer [15].

According to the World Health Organization, recent 2020 survey released by the International Agency for Research on Cancer in 
Globocan 2020. The number of new cancer cases estimated to be $11.7 \%$ of breast cancer, next comes lung cancer $11.4 \%$, colorectal cancer $10 \%$, and the percentage of all the other cancer types were estimated to cover $66.7 \%$. From the data, it is clear that breast cancer is predominant among other types of cancer. Non-performance of the tissue regulatory ends up in the non-controllable multiplication of the normal cells, resulting in the tumour. Cancer can be caused by different factors like mutations in gene level, hereditary, and by carcinogenic factors. [16].

It is also found that hypoxia condition is also related to the advancement and metastasis condition $[17,18]$. It also includes metastasis in breast cancer $[19,20]$. Besides, numerous supporting pieces of evidence are suggesting that tumour metabolism and hypoxia-inducible factor 1a (HIF-1a) regulates an enzyme called carbonic anhydrase (CA IX) and CA-XII. They participate in transferring the tumour progression to a metastasis state [21]. CA is an enzyme with dimeric membrane [22,23]. After recognizing the association and expression of CA IX with the progression of breast cancer and poor treatment, results had turned the interest of targeting the CA IX enzyme in the cancer treatment [24]. CA IX was used as a biomarker of hypoxic tumors and also as a specific antibody and sulfonamide-base molecule for identifying CA IX in vivo studies [25].

Insilco molecular docking had changed the folk medicines, and it became an important tool in drug discovery, compared to the cost spend on a wet-lab method of the drug discovery process, it has reduced the cost and its simple methodology has turned the interest of the academicians [26]. Molecular docking works in the principle of predicting the preferable orientation of ligand with the target molecule, which results in the complex molecule formation. The bond formed between the ligand and the target molecule is due to the binding affinity formed between the two molecules which can be predicted by the scoring functions [27].

In this study, the antioxidant potential of the extracted fucoidan (FE) from the $T$. conoides was determined and the potential of fucoidan to dock with CA IX enzyme was determined to overcome breast cancer through natural medicines without any side effects.

\section{MATERIALS AND METHODOLOGY}

\subsection{Sample Collection}

Turbinaria conoides was collected from Mandapam coastal area, Rameshwaram. The collected sample was cleaned, shade-dried, and then it was further dried using a hot air oven. The dried seaweeds were subjected to a mixer machine. The powdered $T$. conoides sample was stored in an airtight container and used for carrying out further analysis.

\subsubsection{Extraction methodology}

Fucoidan from T. conoides was extracted by the following methodology [28]. $100 \mathrm{~g}$ of powdered T. conoides seaweed sample was mixed with 2.41 of distilled water and it was kept in the shaker for 24 hours. The mixture was subjected to filtration and the filtrate was separated. $150 \mathrm{ml}$ of the filtrate solution was taken and mixed with $1 \%$ chitosan. ( $1 \%$ chitosan was prepared in $1 \%$ acetic acid solution). It was kept for 12 hours in a shaker for proper agitation.
The obtained solution was centrifuged at 1,500 rpm for 5 minutes. The supernatant was removed and the pellet was dried in the hot air oven at $50^{\circ} \mathrm{C} .4 \mathrm{~g}$ of the pellet was obtained and it was dipped in 551 $\mathrm{ml}$ of distilled water. $\mathrm{pH}$ was adjusted to 6 and it was kept for 12 hours. After 12 hours, the mixture was agitated at room temperature for 2 hours and it was filtered. The filtrate was washed with ethanol for 15 minutes, and it was centrifuged and the pellet was removed, air-dried, and it was used for further analysis. The extracted FE was confirmed using standard fucose by high performance liquid chromatography (HPLC) [29]. Then, it was subjected to antioxidant activities to find its antiscanvenging potential.

\subsubsection{Antioxidant activity}

2.1.2.1. Ferric reducing antioxidant power assay (FRAP). Reducing power of the FE was determined according to the method of Oyaizu [30]. $1 \mathrm{ml}$ of the extract with the concentration range $(100,200,300,400$, and $500 \mu \mathrm{g} / \mathrm{ml})$ was mixed with $2.5 \mathrm{ml}$ of phosphate buffer $(0.2 \mathrm{M}, \mathrm{pH} 6.6)$ and $2.5 \mathrm{ml}$ of $1 \%$ potassium hexacyanoferrate (III). After incubation for 20 minutes at $50^{\circ} \mathrm{C}$, $2.5 \mathrm{ml}$ of trichloroacetic acid was added and centrifuged at 3,000 rpm for 10 minutes. To $2.5 \mathrm{ml}$ of the supernatant (upper layer), $2.5 \mathrm{ml}$ of distilled water and $0.5 \mathrm{ml}$ of $\mathrm{FeCl} 3(0.1 \%)$ were added and the absorbance was measured at $700 \mathrm{~nm}$. The reducing power of FE was determined by comparing it with the standard ascorbic acid. Concentration was similar like the test sample was taken.

\subsubsection{Superoxide dismutase (SOD)}

Measurement of superoxide anion scavenging activity was performed based on the method of Nishimiki et al. [31] with slight modifications. The reaction mixture contained $1 \mathrm{ml}$ of nitroblue tetrazolium $(156 \mu \mathrm{M}), 1.0 \mathrm{ml}$ of phosphate buffer $(100 \mathrm{mM}, \mathrm{pH}$ $7.4)$, and $1 \mathrm{ml}$ of nicotinamide adenine dinucleotide $(468 \mu \mathrm{M})$ in $100 \mathrm{mM}$ phosphate buffer along with $0.1 \mathrm{ml}$ of test samples with the concentration $(100,200,300,400$, and $500 \mu \mathrm{g} / \mathrm{ml})$. The reaction was started by adding $100 \mu \mathrm{l}$ of phenazinemethosulphate $(60 \mu \mathrm{M}$ PMS) in phosphate buffer and incubated at $25^{\circ} \mathrm{C}$ for 5 minutes. The absorbance was measured at $560 \mathrm{~nm}$ and butylatedhydroxytoluene (BHT) was used as the reference. The percentage of inhibition was calculated as mentioned below.

$\%$ of $\mathrm{SOD}=($ Absorbance of control - Absorbance of test $) /$ Absorbance of control $\times 100$

\subsubsection{Molecular docking}

Fucoidan compound confirmed by HPLC was subjected to target prediction by using the Swiss target server [32] Swiss target prediction server works on the similarity principle according to which, two structurally similar molecules are prone to have similar biological properties [33]. Target molecule carbonic anhydrase IX (CA IX) was retrieved from (PDB ID 2HKF) and the $3 \mathrm{D}$ structures of the fucoidan compound was retrieved from the Pubchem database (CID: 92023653) in sdf format. The chosen ligand was manually prepared using the ligand preparation module of Molegro virtual docker (MVD). CA IX. The binding cavity for the docking process was chosen based not only on the MVD but also based on the binding site information gathered from the crystal structure of CA IX ( PDB 2 HKF) This also correlated 
with active site residue of CA IX has given in the detailed study by Alterio et al. [34]. Initially, the basic physical descriptors like molecular weight, $\log P$, number of hydrogen bond acceptors, and hydrogen-bond donors were calculated for the fucoidan compound using molinspiration. The chosen compound was found to abide by the "Lipinski's Rule" of " 5 " filter thus it was taken for further steps. Molecular docking was performed between fucoidan and the target molecule human CA IX (PDB ID 2HKF). Out of many docking poses, only those poses with the highest moldock score and relatively good hydrogen bond interaction were chosen. The intact protein structure was loaded onto the MVD platform for the docking process. The potential binding site also referred as cavities or active site was identified using the build-in cavity detection algorithm of MVD. The search algorithm is taken as Moldock SE and the number of runs taken are 10 and the max iteration was 2,000 with the population size of 50 with an energy threshold of 100. At each step least "minute" torsions/translations/ rotations were tested and the one that gives the lowest energy was chosen. After the docking stimulation was over, the poses which were generated process, the poses which were generated were sorted by moldock score. The chosen ligands were manually prepared using the ligand preparation module of MVD. Bond order and hybridization assigned wherever missing and flexible torsion and ligands were deducted. The target protein structures were prepared after careful removal of heteroatoms and water molecules and its electrostatic surface was generated. The grid resolution was set at $0.3 \AA$. The maximum interaction was set at 1,500 and the maximum population size 50 .

\section{RESULTS AND DISCUSSION}

\subsection{Ferric Reducing Antioxidant Power Assay}

The ferric reducing power of the FE was analyzed and determined by the spectrophotometer at $700 \mathrm{~nm}$ and the $\%$ of inhibition concentration FE was found to increase with the increase in the concentration in a dose-dependent manner, and the optical density (OD) values were found to be $0.016,0.029,0.047,0.063$, and 0.081 with increasing concentration ranging from 100, 200, 300, 400 , and $500 \mu \mathrm{g} / \mathrm{ml}$. The highest ferric reducing power of FE was obtained at $500 \mu \mathrm{g} / \mathrm{ml}$ compared to the standard ascorbic acid, and the OD value for standard was found to be $0.012,0.026,0.089$, 0.051 , and 0.062 for the respective concentration ranging from 100 to $500 \mu \mathrm{g} / \mathrm{ml}$. The results were shown in (Fig. 1) in this assay the reducing power of the FE was determined by the change in the colour shade. Solution colour was found to change from green to blue shade, with the increase in the concentration. This acts as an indicator of the ferric reducing capacity of the fucoidan extracted from $T$. conoides.

\subsubsection{Superoxide dismutase}

Superoxide radicals can cause severe damage to cells and tissues through forming other ROS such as single oxygen and hydroxyl radicals [35]. The FE scavenging effect of the superoxide radicals was measured in the spectrophotometer at $540 \mathrm{~nm}$ at varying concentrations ranging from $100,200,300,400,500 \mu \mathrm{g} / \mathrm{ml}$, and the $\%$ of inhibition of the fucoidan was found to be $17.01 \%, 32.78 \%$, $49.88 \%, 67.13 \%$, and $82.41 \%$ for the respective concentration with

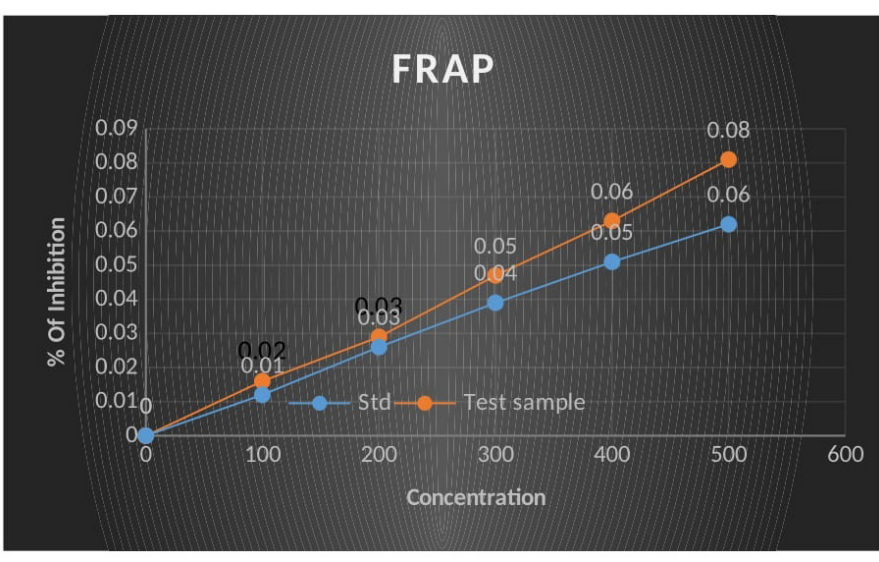

Figure 1: Graphical representation of the FE inhibiting ferric reducing capacity.

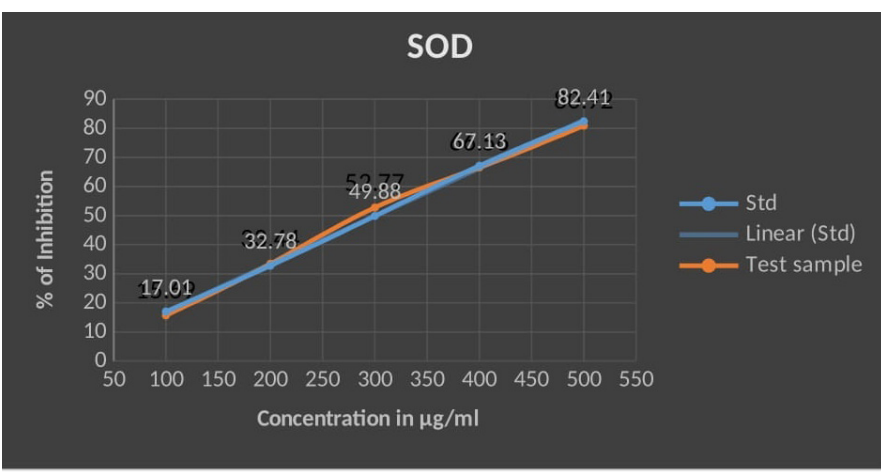

Figure 2: Graphical representation of FE inhibiting the scavenging effect of the sodium dismutase assay.

IC50 values of $300.957 \mu \mathrm{g} / \mathrm{ml}$ for the FE. There was an increase in the inhibition with an increase in the concentration in a dosedependent manner and for the standard \% of inhibition was found to be $15.69 \%, 33.44 \%, 52.77 \%, 66.56 \%$, and $84.06 \%$ with an IC 50 value of $300.758 \mu \mathrm{g} / \mathrm{ml}$ for the standard BHT and the results are shown in Figure 2, the FE exhibited significant scavenging effects at all tests concentrations. The scavenging effect shows a significant increase with the increase in the concentration.

\subsubsection{Results from molecular dynamics docking}

Fucoidan compound retrieved from Pubchem CID 92023653 was docked with the 3D structure of human carbonic anhydrase (CAIX PDB ID 2HKF) out of the many docking poses, only those which possess the highest moldock score and relatively good hydrogen bond interaction was chosen and the docking results were tabulated (Table 1).

Fucoidan was found to form multiple hydrogen bonds (8) with the active site of CA IX the interacting amino acids are found to be ARG52, ARG50, TYR101, GLY102, ASN35, and ALA33. Fucoidan also formed vanderwaal interactions with SER96, PHE101. These interactions are depicted in Figure 3. In 1A, secondary structure view of human carbonic anhydrase IX (PDB ID:2HKF) in complex with L-fucoidan which is shown in the green stick model. The zoomed region $1 \mathrm{~B}$ depicts the docking view of L-fucoidan within the active site of human carbonic anhydrase IX 
Santhanam et al.: Molecular docking of fucoidan (a polysaccharide compound Turbinaria conoides) with carbonic

anhydrase IX 2021;9(S1):20-25

Table 1: Molecular docking of the fucoidan with target molecule and its scoring.

\begin{tabular}{|c|c|c|c|c|c|}
\hline Compound name & Pubchem ID & Molgro score & H-band score & No of $\mathrm{H}$ bond & Interactions with amino acids \\
\hline \multirow[t]{5}{*}{ Fucoidan } & 92023653 & -66.5818 & -18.8865 & 8 & GLN39 \\
\hline & & & & & GLN39 \\
\hline & & & & & GLN47 \\
\hline & & & & & GLY4(62) \\
\hline & & & & & LYS44(2) \\
\hline
\end{tabular}

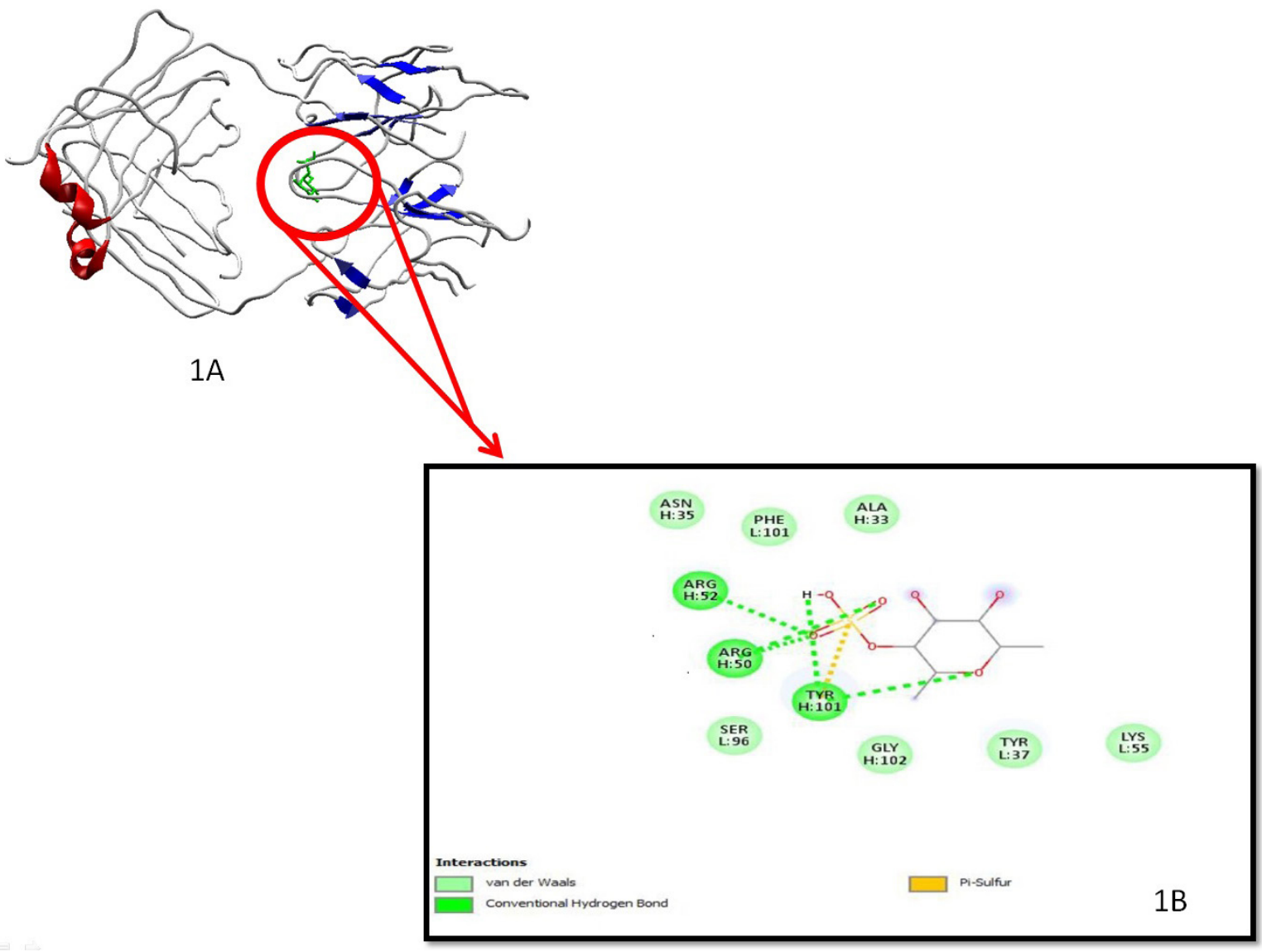

Figure 3: Docking view of L-Fucose within the active site of Human Carbonic anhydrase IX.

where hydrogen bonds are depicted in bright green dotted lines, whereas those amino acids forming van der waals interaction are shown in faded green colour.

The binding affinity of fucoidan with CA IX was compared with the binding affinity of other known inhibitors, such as oxamide. Fucoidan was found to interact with the active site of CA IX forming eight hydrogen bonds, whereas oxamide forms only four hydrogen bonds. The Molgro score of binding was also better for fucoidan when compared with oxamide.

Thus, Fucoidan showed a good binding affinity toward CA IX. That is indicated by the Molgro score of -66.58 and $\mathrm{H}$ bond score of -18.4 more negativity denotes higher affinity.

Cancer remains one of the most lethal diseases worldwide. There is an urgent need for new drugs with novel modes of action, and thus considerable research has been conducted for new anticancer drugs from natural sources, especially plants, microbes, and marine organisms. Marine populations represent reservoirs of novel bioactive metabolites with diverse groups of chemical structures. Extracts taken from marshy flora are used worldwide for their natural medicinal values. It is also recorded that there are around 349 metabolites are present in the marshy floras that are used for their medicinal value [36]; in this study, fucoidan was extracted from $T$. conoides, and the antioxidant activity was obtained with significant results. It is also reported that brown seaweeds gains more attention by the researchers for their highly beneficial effects to the human beings because they possess a good antimicrobial and anti-scavenging activity and it is also reported that ethanol extract $T$. conoides possessed minimum anticancer activity on different types of cancer cells [37].

The chosen antiscavenging activities of FE extracted from $T$. conoides showed a significant result compared to the standard 
Ascorbic acid, in a dose-dependent manner. This reveals that fucoidan has a very good potential in arresting the free radicals and possess the anti-scavenging property in them. Possible antioxidant and cytotoxic activity of fucoidan extracted from other seaweeds like Turbinaria ornata, Padina tetrastromatica, and Sargassumwightii collected from Tamil Nadu southeast coastal region was found to be a rich source of carbohydrates, sulfate, and uronic acid, and the anti-scavenging property of those extracts was due to the presence of fucoidan. This was reported in the earlier findings and it was confirmed and the functional and structural relationship of fucoidan was also correlated [38]. According to the 2018 statistical breast cancer report given by cytecare cancer Hospital, breast cancer was found to overtake cervical cancer. Carbonic anhydrase IX (CA IX) is a tumor-associated, cell-surface glycoprotein [39]; the glycolytic metabolism of cancer was evaluated for many years to describe the fundamental correlation between the glycolytic metabolism of cancer cell growth and progression was evaluated for many years to report on the microenvironment and glycolysis in cancer growth progression [40]. Patrou [41] found that transcription factors of the glycolytic pathway were affected by cell proliferation that was found to be an important feature of carcinogenesis. It is also stated that different types of enzymes are produced in our body based on the type of malignancy, such types of enzymes produced are used as cancer biomarkers. He also reported that the type and the expression of the enzymes vary in different types of cancer cells [42].

It is also found that the expression of CA IX is overexpressed in different types of cancer cells $[43,44]$. In recent research studies, it is also observed that SLC0111 (Ureido- substituted benzenesulfonamide molecule), a potent inhibitor of CA IX which is confirmed as an antitumor agent by phase I clinical studies [45].

It is hard to find the CA IX expression in normal tissues but it could be scarcely found in the lining of stomach epithelial tissues, intestine, and other organs located near the stomach region. More than 1,000 research publications have estimated the current literature biological role of CAIX and it is also used as a biomarker in identifying the different types of cancer. This was very clearly illustrated [46] and because of the above reason, our study was extended to molecular docking using CA IX as a target molecule. Molecular docking is a key tool to find the structure-based drug design in an in-vitro condition and found to show good affinity with the target molecule.

\section{CONCLUSION}

Results obtained from FE extracted from $T$. conoides was found to contain a natural bioactive compound-Fucoidan. We suggest that the results are mainly due to the activity of fucoidan because in our earlier studies [29], the presence of fucoidan was confirmed by HPLC analysis and the presence of fucoidan extract was in higher concentration compared to the other components in the extract. Thus, significant anti-scavenging activities may be due to the presence of fucoidan in the extract. Molecular docking of fucoidan with the target molecule carbonic anhydrase IX (CA IX) formed a good binding site. This indicates that targeting the CA IX enzyme may reduce breast cancer in ethnopharmacological studies, which may reduce the intensity of side effects. It is also found CA IX isoenzyme is found to be a prominent biomarker in liquid biopsy for the detection of solid tumours like breast, lung, and pancreatic [47]. Therefore, CA IX could be a valid molecular target in anticancer treatment. Thus, FE extracted from $T$. conoides can be further extended to in vivo studies to find the preventive action against breast cancer.

\section{ACKNOWLEDGMENT}

The authors acknowledge Dr. Abhinand, Department of Bioinformatics, Sri Ramachandra Institute of Higher Education and Research for helping in docking process.

\section{AUTHOR CONTRIBUTIONS}

All authors made substantial contributions to conception and design, acquisition of data, or analysis and interpretation of data; took part in drafting the article or revising it critically for important intellectual content; agreed to submit to the current journal; gave final approval of the version to be published; and agree to be accountable for all aspects of the work. All the authors are eligible to be an author as per the international committee of medical journal editors (ICMJE) requirements/guidelines.

\section{FUNDING}

There is no funding to report.

\section{CONFLICTS OF INTEREST}

The authors report no financial or any other conflicts of interest in this work.

\section{ETHICAL APPROVAL}

This study does not involve experiments on animals or human subjects.

\section{REFERENCES}

1. Wang ZJ, Zheng L, Yang JM, Kang Y, Park YD. Proteomic analyses for profiling regulated proteins/enzymes by Fucusvesiculosus fucoidan in B16 melanoma cells: a combination of enzyme kinetics functional study. Int J Biol Macromol 2018;112:667-74.

2. Deniaud-Bouët E, Hardouin K, Potin P, Kloareg B, Hervé C. A review about brown algal cell walls and fucose-containing sulfated polysaccharides: cell wall context, biomedical properties and key research challenges. Carbohydr Polym 2017;175:395-408.

3. Sanjeewa KKA, Lee JS, Kim WS, Jeon YJ. The potential of brownalgae polysaccharides for the development of anticancer agents: an update on anticancer effects reported for fucoidan and laminaran. Carbohydr Polym 2017;177:451-9.

4. Omar HE, Eldien HM, Badary MS, Al-Khatib BY, AbdElgaffar SK. The immunomodulating and antioxidant activity of fucoidan on the splenic tissue of rats treated with cyclosporine A. J Basic Appl Zool 2013;66:243-54.

5. Ngo DH, Kim SK. Sulfated polysaccharides as bioactive agents from marine algae. Int J Biol Macromol 2013;62:70-5.

6. Raghavendran HR, Srinivasan P, Rekha S. Immunomodulatory activity of fucoidan against aspirin-induced gastric mucosal damage in rats. Int Immunopharmacol 2011;11(2):157-63; doi:10.1016/j. intimp.2010.11.002.

7. Synytsya A, Bleha R, Synytsya A, Pohl R, Hayashi K, Yoshinaga K, et al. Mekabufucoidan: structural complexity and defensive effects against avian influenza A viruses. Carbohydr Polym 2014;111: $633-44$. 
8. Balboa EM, Conde E, Moure A, Falqué E, Domínguez H. In vitro antioxidant properties of crude extracts and compounds from brown algae. Food Chem 2013;138(Suppl 2-3):1764-85.

9. Liu L, Heinrich M, Myers S, Dworjanyn SA. Towards a better understanding of medicinal uses of the brown seaweed Sargassum in traditional Chinese medicine: a phytochemical and pharmacological review. J Ethnopharmacol 2012;142:591-619.

10. Chakraborty K, Praveen NK, Vijayan KK, SydaRao G. Evaluation of phenolic contents and antioxidant activities of brown seaweeds belonging to Turbinaria spp. (Phaeophyta, Sargassaceae) collected from Gulf of Mannar. Asian Pac J Trop Biomed 2013;3(1):8-16.

11. Finkel T, Holbrook NJ. Oxidants, oxidative stress and the biology of aging. Nature 2000; 408:239-47.

12. Wiseman H, Halliwell B. Damage to DNA by reactive oxygen and nitrogen species: role ininflammatory disease and progression to cancer. Biochem J 1996;313:17-29.

13. Lee SE, Hwang HJ, Ha JS, Jeong HS, Kim JH. Screening of medicinal plant extracts for antioxidant activity. Life Sci 2003;73:167-79.

14. Schinella GR, Tournier HA, Prieto JM, de Buschiazzo PM, Rios JL. Antioxidant activity of anti-inflammatory plant extracts. Life Sci 2002;70:1023-33.

15. Di Matteo V, Esposito E. Biochemical and therapeutic effects of antioxidants in the treatment of Alzheimer's disease, Parkinson's disease, and amyotrophic lateral sclerosis. Curr Drug Target CNS Neurol Disord 2003;2:95-107.

16. Mahdi-Pour B, Jothy SL, Latha LY, Chen Y, Sasidharan S. Antioxidant activity of methanol extracts of different parts of Lantana camara. Asian Pac J Trop Biomed 2012;2(12):960-5.

17. Reddy ARK, Grace JR. Anticancer activity of methanolic extracts of selected mangrove plants. Int J Pharm Sci Res 2007;7(9):3852-6.

18. Brahimi-Horn MC, Chiche J, Pouyssegur J. Hypoxia and cancer. J Mol Med 2007;85:1301-7.

19. Chaudary N, Hill RP. Hypoxia and metastasis. Clin Cancer Res 2007;13:1947-9.

20. Finak G, Bertos N, Pepin F, Sadekova S, Souleimanova M, Zhao H, et al. Stromal gene expression predicts clinical outcome in breastcancer. Nat Med 2008;14:518-27.

21. Kroemer G, Pouyssegur J. Tumor cell metabolism: cancer's Achilles'heel. Cancer Cell 2008;13:472-82.

22. Chaudary N, Hill RP. Hypoxia and metastasis in breast cancer. Breast Dis 2006;26:55-64.

23. Alterio V, Hilvo M, Di Fiore A, Supuran CT, Pan P, Parkkila S, et al. Crystal structure of the catalytic domain of the tumor-associated human carbonic anhydrase IX. Proc Natl Acad Sci U S A 2009;106:16233-8.

24. Supuran CT. Carbonic anhydrases: novel therapeutic applications for inhibitors and activators. Nat Rev Drug Discov 2008;7:168-81.

25. Akurathi V, Dubois L, Lieuwes NG, Chitneni SK, Cleynhens BJ, Vullo D, et al. Synthesis and biological evaluation of a 99mTc-labelled sulfonamide conjugate for in vivo visualization of carbonic anhydrase IX expression in tumor hypoxia. Nucl Med Biol 2010;37:557-64.

26. Berry M, Fielding B, Gamieldien J. Practical considerations in virtual screening and molecular docking. In: Tran QN, Arabnia HR (eds.). Emerging trends in computational biology, bioinformatics, and systems biology. National Center for Biotechnology Information, U.S. National Library of Medicine, Rockville Pike, Bethesda MD, pp 487$502,2015$.

27. Lengauer T, Rarey M. Computational methods for biomolecular docking. Curr Opin Struct Biol 1996;6(3):402-6; doi:10.1016/S0959440X(96)80061-3.

28. Chen X, Xing R, Yu H, Liu S, Li P. A new extraction method of fucoidan from the soaked water of brown seaweed (Laminaria japonica) Desalin Water Treat 2012;40:204-8.

29. Santhanam RC, Yahoob SAM, Venkatraman A. Antiscavenging property and characterization using HPLC of the fucoidan extracted from Turbinaria conoides. Int J Sci Technol Res 2019;8(11):2201-6.

30. Oyaizu M. Studies on product of browning reaction prepared from glucose amine. Jap J Nutr 1986;44:307315.
31. Nishimiki M, Rao NA, Yagi K. The occurrence of super-oxide anion in the reaction of reduced phenazinemethosulfate and molecular oxygen Biochem Biophys Res Commun1972;46:849-53.

32. Gfeller D, Grosdidier A, Wirth M, Daina A, Michielin O, Zoete V. Swiss target prediction: a web server for target prediction of bioactive small molecules. Nucleic Acids Res 2014;42(Web Server issue):W32-8.

33. Daina A, Michielin O, Zoete V. Swiss target prediction: updated data and new features for efficient prediction of protein targets of small molecules. Nucleic Acids Res. 2019;47(W1):W357-64.

34. Alterio V, Hilvo M, Di Fiore A, Supuran CT, Pan P, Parkkila S, et al. Crystal structure of catalytic domain of the tumor-associated human carbonic anhydrase IX. Proc Natl Acad Sci [Internet] 2009;106(38):16233-8

35. Dahl MK, Richardson T. Photo generation of superoxide anion in serum of bovine milk and in model systems containing riboflavin and amino acids. J Dairy Sci 1978;61:400-7.

36. Bandaranayake WM. Bioactivities, bioactive compounds and chemical constituents of mangrove plants. Wetlands Ecol Manage 2002;10(6):421-52.

37. Ponnan A, Ramu K, Marudhamuthu M, Marimuthu R, Siva K, Kadarkarai M. Antibacterial, antioxidant and anticancerproperties of Turbinaria conoides (J. Agardh) Kuetz clinical phytoscience. Clin Phytosci 2017;3:5; doi:10.1186/s40816-017-0042-y pg 2-10.

38. Gopidas SK, Subramani N. In vitro antioxidant and cytotoxic properties of fucoidan from three Indian brown seaweeds, Asian J Pharm Clin Sci 2019;12(9):99-105.

39. Pastorek, J, Pastorekova S, Callebaut I, Mornon JP, Zelník V, Opavský $\mathrm{R}$, et al. Cloning and characterization of $\mathrm{MN}$, a human tumorassociated protein with a domain homologous to carbonic anhydrase and a putative helix-loop-helix DNA binding segment. Oncogene 1999;9:2877-88.

40. Jang M, Kim SS, Lee J. Cancer cell metabolism, implication for therapeutic targets, Exp Mol Med 2013;45:e45.

41. Petrou A, Geronikaki A, Terzi E, Guler OO, Tuccinardi T, Supuran CT. Inhibition of carbonic anhydrase isoforms I II, IX and XII with secondary sulfonamides incorporating benzothiazole scaffolds. J Enzyme Inhib Med Chem 2016;31:1306-11.

42. Neri D, Supuran CT. Interfering with $\mathrm{pH}$ regulation in tumour as therapeutic strategy. Nat Rev Drug Discov 2011;10:767-77.

43. Winum JY, Rami M, Scozzafava A, Montero JL, Supuran C. Carbonic anhydrase IX: a new druggable target for the design of antitumor agents. Med Res Rev 2008;28:445-63.

44. Winum JY, Pastorekova S, Jakubickova L, Montero JL, Scozzafava A, Pastorek J, et al. Carbonic anhydrase inhibitors synthesis and inhibiyion of cytosolic/tumour- associated carbonic anhudraseisozyme I,II, and IX with bis-sulfamates. Bioorg Med Chem Lett 2005;15:57984.

45. Lomelino CL, Mahon BP, McKenna R, Carta F, Supuran CT. Kinetic and X-ray crystallographic investigations on carbonic anhydrase isoforms I, II, IX and XII of a thioureido analog of SLC-0111. Bioorg Med Chem 2016;24:976-81.

46. Pastorek J, Pastorekova S. Hypoxia-induced carbonicanhydrase IX as a target for cancer therapy: from biology to clinicaluse. Semin Cancer Biol 2015;31:52-64.

47. Ozensoy Guler O, Supuran CT, Capasso C. Carbonic anhydrase IX as a novel candidate in liquid biopsy. J Enzyme Inhib Med Chem 2020;35:255-60; doi:10.1080/14756366.2019.1697251.

\section{How to cite this article:}

Chitra SR, Yahoob SAM, Anuradha V. Molecular docking of fucoidan (a polysaccharide compound Turbinaria conoides) with carbonic anhydrase IX-A potent target molecule in breast cancer. J Appl Biol Biotech 2021;9(S1):20-25. 